\title{
Spectrum of lesions visualized in cardiac magnetic resonance imaging in COVID-19-related myocarditis: Findings from a pilot study of the TRICITY-CMR trial
}

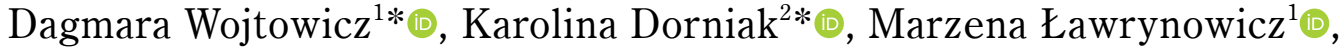 \\ Joanna Rejszel-Baranowska ${ }^{1}\left[{ }^{0}\right.$, Jadwiga Fijałkowska ${ }^{3}$, Dorota Kulawiak-Gałąska ${ }^{4}$, \\ Edyta Szurowska ${ }^{3}$, Marek Koziński ${ }^{1}$ (D) \\ ${ }^{1}$ Department of Cardiology and Internal Diseases, Institute of Maritime and Tropical Medicine, \\ Medical University of Gdansk, Gdynia, Poland \\ ${ }^{2}$ Department of Noninvasive Cardiac Diagnostics, Medical University of Gdansk, \\ University Clinical Center in Gdansk, Poland \\ ${ }^{3}$ Department of Radiology II, Medical University of Gdansk, University Clinical Center in Gdansk, Poland \\ ${ }^{4}$ Department of Radiology, Medical University of Gdansk, University Clinical Center in Gdansk, Poland
}

Myocardial injury with an elevated concentration of cardiac troponins is a prevalent condition associated with increased in-hospital mortality in patients with coronavirus disease-2019 (COVID-19) [1]. Myocarditis may be the underlying pathology in some patients with COVID-19-related myocardial injury. Additionally, a substantial proportion of patients who have recovered from COVID-19 pneumonia present with persistent symptoms indicating sustained cardiac involvement. Therefore, it is of major clinical importance to investigate the association between cardiac symptoms and possible myocardial lesions in post-COVID-19 patients.

Cardiac magnetic resonance (CMR) is considered the gold standard to assess cardiac morphology and function. Moreover, unlike other imaging modalities, it allows for detailed tissue characterization.

In this research letter, we report on the findings of a pilot study including patients with a history of recent COVID-19 pneumonia confirmed by a positive real-time polymerase chain reaction test and referred to our outpatient post-COVID-19 cardiology clinic. This paper is focused on types of lesions and their prevalence observed in CMR imaging. The TRICITY-CMR trial was designed as a prospective, cohort study including patients presenting with symptoms suggesting the involvement of the heart (e.g., chest pain, palpitations, dyspnea). In all the study participants, extracardiac etiology of symptoms seemed unlikely based on available test results. We excluded patients with any known previous cardiac pathology except essential hypertension as well as those with contraindications for CMR imaging. Clinical data and CMR imaging were analyzed. The study protocol was approved by the local ethics committee, and subsequently all patients provided informed written consent. Patients were recruited between December 2020 and March 2021. CMR was performed on a 1.5-T scanner (Magnetom Aera or Magnetom Sola, Siemens AG, Erlangen, Germany), and the protocol included standard long-axis and short-axis cine series for anatomy and functional assessment, followed by cardiac parametric mapping (MyoMaps, Siemens Healthineers, Erlangen, Germany) and late gadolinium enhancement (LGE) (free breathing phase-sensitive inversion recovery,

Address for correspondence: Dagmara Wojtowicz, MD, PhD, Department of Cardiology and Internal Diseases, Institute of Maritime and Tropical Medicine, Medical University of Gdansk, ul. Powstania Styczniowego 9B, 81-519 Gdynia, Poland, tel: +485869984 06, e-mail: dagmara.wojtowicz@gumed.edu.pl

Received: 14.05.2021 Accepted: 23.09.2021 Early publication date: 27.10.2021

*Equal contributors.

This article is available in open access under Creative Common Attribution-Non-Commercial-No Derivatives 4.0 International (CC BY-NC-ND 4.0) license, allowing to download articles and share them with others as long as they credit the authors and the publisher, but without permission to change them in any way or use them commercially. 


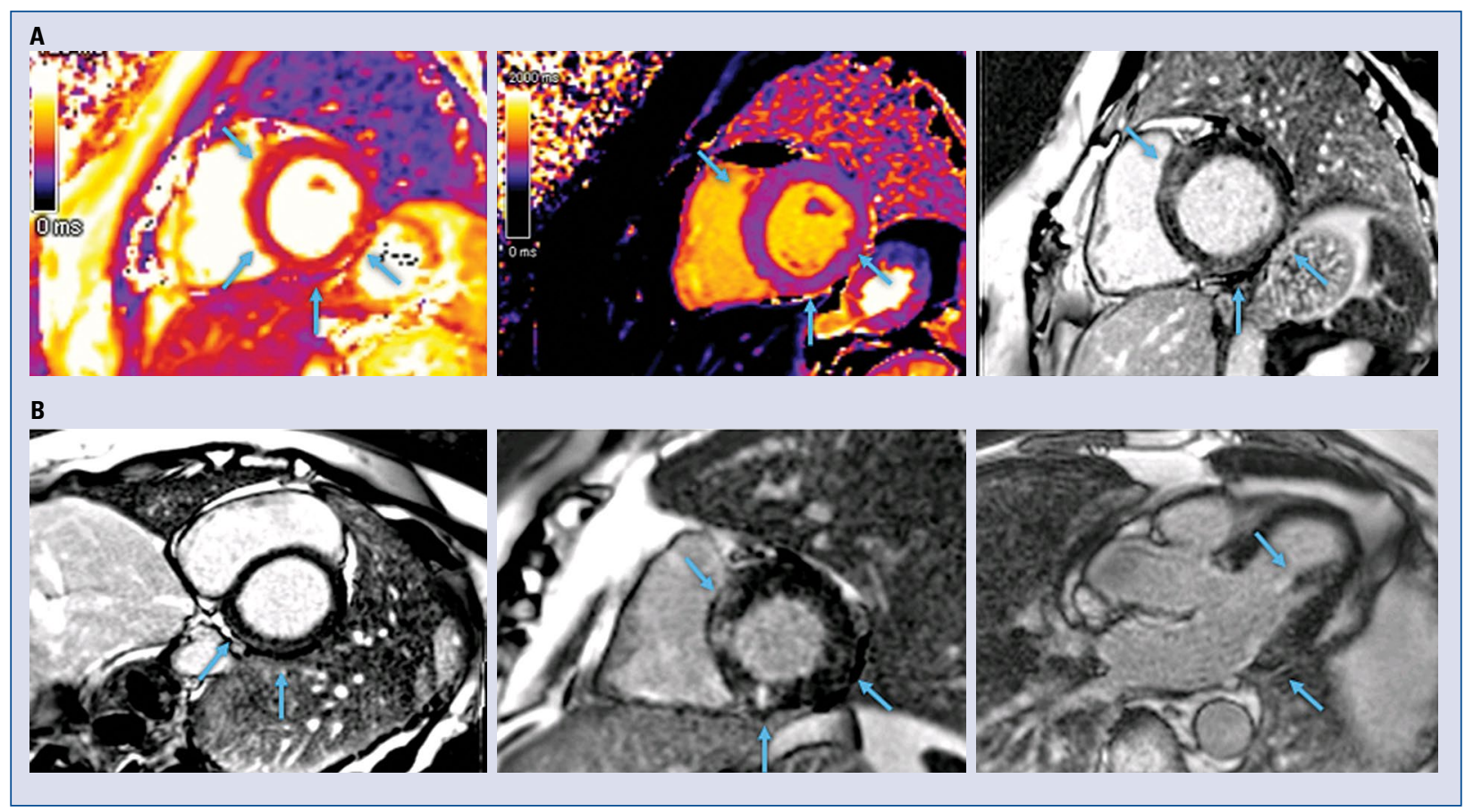

Figure 1. Typical lesions found in cardiac magnetic resonance examination in patients with a history of recent COVID-19 pneumonia and symptoms suggesting cardiac involvement. A. Locally (blue arrows) increased T2 (left) and T1 (middle) relaxation times pointing out areas of acute injury/ongoing inflammation, paralleled by subtle intramyocardial areas of irreversible damage (inflammatory necrosis/fibrosis) as shown by late gadolinium enhancement (LGE) (right) in a 63-year-old patient about 6 weeks post COVID-19 pneumonia. Global T2 relaxation time was 46 ms (the institutional reference range: $39-49 \mathrm{~ms}$ ) with local (arrows) increase (segmental ROI average values T2 = $52 \mathrm{~ms}$ in the basal septal, T2 = $52 \mathrm{~ms}$ in the basal inferior, and $53 \mathrm{~ms}$ in the basal inferolateral segments). Global T1 value was $994 \mathrm{~ms}$ (the institutional reference range: $951-1035 \mathrm{~ms}$ ) with local increase in the septum (1065 ms) as well as inferior (1050 ms) and inferolateral segments (1056 ms); B. Examples of LGE patterns in the study group (blue arrows); Left: Subtle subepicardial LGE in the basal inferior and/or inferolateral segment, representing the most common LGE pattern in our post-COVID cohort; Middle: Intramyocardial areas of LGE in the basal septal, inferior, and inferolateral segments; Right: Long-axis 3-chamber plane in the same patient, showing involvement of the basal inferolateral segment and the posterior papillary muscle.

motion-corrected [PSIR MOCO] sequence) for tissue characterization [2].

Fifty consecutive patients experiencing persistent cardiac symptoms after recovery from COVID-19 were included in the study. The mean age of patients was $47.3 \pm 10.1$ (range 27-69) years, and $40 \%(n=20)$ were men. Most of the patients reported dyspnea $(50 \% ; \mathrm{n}=25)$ or fatigue $(36 \% ; \mathrm{n}=18)$ as the predominant complaint. Nearly one-third of the study participants (30\%; $\mathrm{n}=15$ ) had a previous history of hypertension, and $14(28 \%)$ individuals were obese. The mean time from the diagnosis of COVID-19 infection to the CMR examination was $51.5 \pm 28.0$ (range 11-113) days. Twenty-one (42\%) study participants required hospitalization during the acute phase of COVID-19 infection. Among them, 15 (71\%) required supplemental oxygen therapy, $5(24 \%)$ received remdesivir, $15(71 \%)$ steroids, and $3(14 \%)$ were treated with convalescent plasma. Thirty-four $(68 \%)$ patients were qualified as moderately ill and $16(32 \%)$ as severely ill.

Cardiac involvement was confirmed by CMR in $30(60 \%)$ patients. Reduced left ventricular (LV) systolic function according to institutional thresholds based on the literature [3] (i.e., LV ejection fraction $<57 \%)$ was found in $4(8 \%)$ patients. None of the patients had reduced right ventricular (RV) systolic function (i.e., RV ejection fraction $<52 \%$ in men and $<51 \%$ in women) [3].

Late gadolinium enhancement was present in $30(60 \%)$ patients. In this sub-group, $60 \%(\mathrm{n}=18)$ had at least 2 -segment involvement. Three-segment involvement was seen in $4(13 \%)$ cases and $3(10 \%)$ patients had 4-segment involvement. Most LGE lesions were located at inferolateral $(76 \%$, $\mathrm{n}=23)$ and inferior $(43 \%, \mathrm{n}=13)$ segments at base. Additionally, among patients with LGE, in 
2 cases markedly elevated native $\mathrm{T} 1$ and $\mathrm{T} 2$ values were shown, suggestive of ongoing myocardial inflammation. Small pericardial effusion was found in 1 patient. Figure 1 illustrates typical CMR findings noted in our patients.

Importantly, our study demonstrated abnormal CMR findings in the majority of symptomatic patients with recent COVID-19 pneumonia. The most common finding was LGE, predominantly located in the basal inferolateral or inferior segments. A relatively low prevalence of active myocardial inflammation with $\mathrm{T} 1$ and $\mathrm{T} 2$ myocardial mapping was revealed.

Our findings correspond with a previously reported high incidence of myocardial injury secondary to COVID-19 infection [1, 4]. Puntmann et al. [5], in their study of 100 unselected patients after recent COVID-19 pneumonia using CMR imaging, showed cardiac involvement in $78 \%$ of participants and ongoing myocardial inflammation in $60 \%$ of cases. These findings were independent of preexisting conditions, severity and overall course of acute illness, and time from the original diagnosis. Positive LGE with patterns typically occurring in myocarditis was described in prior case reports and observational studies in post-COVID-19 patients $[5,6]$. It is worth emphasizing that the presence of LGE is considered a strong predictor of adverse clinical outcome [7]. Additionally, recent CMR mapping techniques enable quantitative detection of myocardial edema, inflammation, or diffuse fibrosis. In our study group, increased native $\mathrm{T} 1$ and $\mathrm{T} 2$ values suggestive of ongoing myocarditis were observed in $2(4 \%)$ cases, similar to a previous report by Brito et al. [8] and in contrast with the aforementioned study by Puntmann et al. [5]. These apparent discrepancies in the literature should be analyzed with all due consideration regarding cohort characteristics (e.g., age, co-morbidities, disease severity, time from diagnosis). This also underlines the role of the parametric mapping sequences because they quantitatively assess ongoing inflammation and can shed light on the evolution of COVID-19-related myocardial injury. It should be emphasized, however, that mapping techniques are intrinsically complex and can be methodologically challenging. Therefore, to provide reliable insight, they must be utilized according to the guidelines, and institution-specific reference ranges should be established [9]. A prior study indicated that native $\mathrm{T} 1$ and myocardial extracellular volume values are independent risk factors of adverse clinical outcomes in dilated cardiomyopathy [10].
The results of this pilot study indicate the necessity of continued cardiological evaluation of patients with persistent symptoms of possible cardiac origin after recovery from COVID-19 pneumonia. Our findings confirm that persistence of cardiac symptoms after COVID-19 recovery may be related to the heart involvement. Future research is needed to determine the potential clinical significance of CMR findings observed in post-COVID-19 patients.

\section{Conflict of interest: None declared}

\section{References}

1. Shi S, Qin Mu, Shen Bo, et al. Association of cardiac injury with mortality in hospitalized patients with COVID-19 in wuhan, china. JAMA Cardiol. 2020; 5(7): 802-810, doi: 10.1001/jamacardio.2020.0950, indexed in Pubmed: 32211816.

2. Kellman P, Arai AE. Cardiac imaging techniques for physicians: late enhancement. J Magn Reson Imaging. 2012; 36(3): 529-542, doi: 10.1002/jmri.23605, indexed in Pubmed: 22903654 .

3. Kawel-Boehm N, Maceira A, Valsangiacomo-Buechel ER, et al. Normal values for cardiovascular magnetic resonance in adults and children. J Cardiovasc Magn Reson. 2015; 17: 29, doi: 10.1186/s12968-015-0111-7, indexed in Pubmed: 25928314.

4. Inciardi RM, Lupi L, Zaccone G, et al. Cardiac involvement in a patient with coronavirus disease 2019 (COVID-19). JAMA Cardiol. 2020; 5(7): 819-824, doi: 10.1001/jamacardio.2020.1096, indexed in Pubmed: 32219357.

5. Puntmann VO, Carerj ML, Wieters I, et al. Outcomes of cardiovascular magnetic resonance imaging in patients recently recovered from coronavirus disease 2019 (COVID-19). JAMA Cardiol. 2020; 5(11): 1265-1273, doi: 10.1001/jamacardio.2020.3557, indexed in Pubmed: 32730619.

6. Gravinay P, Issa N, Girard D, et al. CMR and serology to diagnose COVID-19 infection with primary cardiac involvement. Eur Heart J Cardiovasc Imaging. 2021; 22(1): 133, doi: 10.1093/ehjci/ jeaa169, indexed in Pubmed: 32556106.

7. Becker MAJ, Cornel JH, van de Ven PM, et al. The prognostic value of late gadolinium-enhanced cardiac magnetic resonance imaging in Nonischemic dilated cardiomyopathy: a review and meta-analysis. JACC Cardiovasc Imaging. 2018; 11(9): 1274-1284, doi: 10.1016/j.jcmg.2018.03.006, indexed in Pubmed: 29680351.

8. Brito D, Meester S, Yanamala N, et al. High prevalence of pericardial involvement in college student athletes recovering from COVID-19. JACC Cardiovasc Imaging. 2021; 14(3): 541-555, doi: 10.1016/j.jcmg.2020.10.023, indexed in Pubmed: 33223496.

9. Messroghli DR, Moon JC, Ferreira VM, et al. Clinical recommendations for cardiovascular magnetic resonance mapping of T1, T2, T2* and extracellular volume: A consensus statement by the Society for Cardiovascular Magnetic Resonance (SCMR) endorsed by the European Association for Cardiovascular Imaging (EACVI). J Cardiovasc Magn Reson. 2017; 19(1): 75, doi: 10.1186/s12968-017-0389-8, indexed in Pubmed: 28992817.

10. Barison A, Del Torto A, Chiappino S, et al. Prognostic significance of myocardial extracellular volume fraction in nonischaemic dilated cardiomyopathy. J Cardiovasc Med (Hagerstown). 2015; 16(10): 681-687, doi: 10.2459/JCM.0000000000000275, indexed in Pubmed: 26090916. 